\title{
Time to treatment initiation and outcomes in high-grade glioma patients in rehabilitation: a retrospective cohort study
}

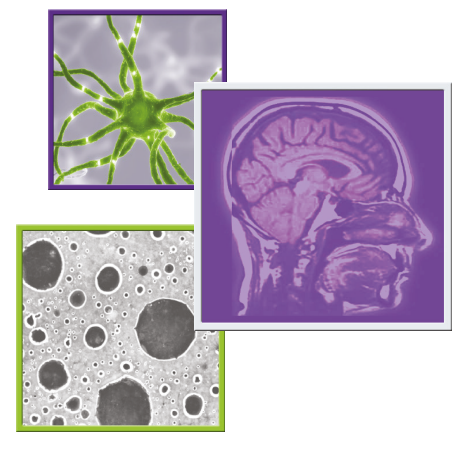

\author{
Kwanza T Warren*,1(D), Linxi Liu², Yang Liư ${ }^{3}$, Myla S Strawderman ${ }^{4}$, Ali H Hussain ${ }^{5}$, \\ Heather M Ma ${ }^{6}$, Michael T Milano ${ }^{7,8}$, Nimish A Mohile ${ }^{8,9}$ \& Kevin A Walter ${ }^{3,8,10}$ \\ ${ }^{1}$ Department of Surgery, New York Presbyterian-Columbia University Medical Center, New York, NY 10032, USA \\ ${ }^{2}$ Department of Public Health Sciences, University of Rochester Medical Center, Rochester, NY 14642, USA \\ ${ }^{3}$ Department of Neurosurgery, University of Rochester Medical Center, Rochester, NY 14642, USA \\ ${ }^{4}$ Department of Biostatistics \& Computational Biology, University of Rochester Medical Center, Rochester, NY 14642, USA \\ ${ }^{5}$ Department of Imaging Sciences, University of Rochester Medical Center, Rochester, NY 14642, USA \\ ${ }^{6}$ Department of Physical Medicine \& Rehabilitation, University of Rochester Medical Center, Rochester, NY 14642, USA \\ ${ }^{7}$ Department of Radiation Oncology, University of Rochester Medical Center, Rochester, NY 14642, USA \\ ${ }^{8}$ University of Rochester Medical Center-Wilmot Cancer Institute, Rochester, NY 14642, USA \\ ${ }^{9}$ Department of Neurology, University of Rochester Medical Center, Neuro-Oncology, Rochester, NY 14642, USA \\ ${ }^{10}$ Department of Orthopaedics, University of Rochester Medical Center, Rochester, NY 14642, USA \\ *Author for correspondence: kw2922@cumc.columbia.edu
}

\begin{abstract}
Aims: To investigate wait time (WT) for chemoradiation and survival in post-op high-grade glioma (HGG) patients admitted to inpatient rehabilitation compared with those discharged home. Materials \& methods: A total of $291 \mathrm{HGG}$ patients (14.4\% grade III and $84.9 \%$ grade IV) were included in this retrospective cohort study. Patients were grouped by disposition following surgery. Results: Median length of stay was longer in acute inpatient rehabilitation facility (AIRF) patients (10d) compared with patients discharged home (3d). AIRF admission was associated with higher odds of excessive treatment delay. Median survival for AIRF patients less than for patients discharged home (42.9 vs 72.71 weeks). WT was not associated with survival even after adjusting for prognostic factors. Conclusion: HGG patients discharged to rehabilitation facilities have longer length of stay, longer WT and shorter survival compared with patients discharged home.
\end{abstract}

First draft submitted: 28 July 2020; Accepted for publication: 16 September 2020; Published online: 28 October 2020

Keywords: chemoradiation $\bullet$ glioblastoma $\bullet$ high-grade glioma $\bullet$ rehabilitation $\bullet$ timing $\bullet$ wait time

High-grade gliomas (HGGs) account for approximately $80 \%$ of all malignant primary brain tumors with the most common being glioblastoma (GBM) and anaplastic astrocytoma at 46.1 and 5.1\%, respectively [1]. The current standard of care that provides the greatest life expectancy was described in the 2005 European Organization for Research and Treatment of Cancer and National Cancer Institute of Canada trial and includes maximal safe tumor resection followed by concurrent radiation and temozolomide and subsequent adjuvant temozolomide [2]. Still, the prognosis for HGG is poor, with 5-year survival ranging from 5.1\% for GBM to $27.9 \%$ for anaplastic astrocytoma [1].

When patients undergo maximal safe tumor resection, they are often discharged to different facilities based on their needs and caregiver support [3]. As patients with brain tumors often have disabilities similar to those who suffered stroke, many of these patients benefit from acute inpatient rehabilitation [4]. Several studies have demonstrated the benefit of rehabilitation on the functional status of patients with brain tumors [5-8] and some studies have even correlated rehabilitation with increased survival [4,9]. However, patients who are admitted to acute inpatient rehabilitation following surgical resection are often ineligible to receive anti-tumor cancer treatment while in a rehabilitation facility and may face delays in treatment.

Studies investigating the optimal timing of chemoradiation following surgical resection in HGG have yielded conflicting results [10]. Some studies have found no association between time from surgical resection to treatment

Future $\because$ Medicine 
initiation (wait time: WT) and overall survival (OS) [11-13], while others have described an inverse relationship between WT and OS [14-16]. Other studies have described a potential benefit to a slight delay in treatment, though a mechanism has not been well established $[17,18]$. However, each of these studies has several limitations based on the differing methodology and retrospective nature of the analyses.

In current practice, the timing of the initiation of chemoradiation following surgery is dependent on clinician judgment, with most US-based HGG patients starting chemoradiation within 4-6 weeks after surgery [19]. Most clinical trials for newly diagnosed GBM expect therapy to be initiated no later than 6 weeks from surgery. Despite the lack of definitive evidence, the prospect of entering a rehabilitation program that may result in a delay in treatment of such an aggressive tumor can serve as a deterrent for patients. We aimed to retrospectively study the outcomes of patients entering inpatient rehabilitation to better inform future treatment guidelines for these patients. The purpose of this study is to investigate the time between surgery, the initiation of chemoradiation and OS in patients who are admitted to rehabilitation facilities when compared with patients who are discharged home after surgery. We hypothesize that patients who are admitted to rehabilitation will have to wait longer to begin anti-cancer treatment.

\section{Materials \& methods}

Selection of patients

All patients age $\geq 18$ years with a pathologically confirmed WHO Grade III or IV glioma treated at our institution from 2011 to 2017 were identified. Patients were included if the electronic health record had information regarding disposition from the hospital stay during which their initial surgical procedure was performed (Supplementary Figure 1). This study was approved by our institution's Research Study Review Board with a waiver of informed consent.

\section{Data extraction}

Demographic factors, tumor histology, tumor location, extent of resection, American Society of Anesthesiologists (ASA) classification, the length of the initial hospital stay (length of stay: LOS), WT (time from surgical procedure to the first date of chemoradiation), OS and disposition from initial hospital stay were collected. If patients had a biopsy followed by resection within a week, the date of the second procedure was used as the start time point of WT. For patients who did not start chemotherapy and radiation on the same day, the earliest date of treatment was chosen to calculate WT. OS was defined starting at the first day of chemoradiation to the date of death or last follow-up to allow for statistical assessment of a relationship between WT and OS. Clinical criteria for discharge to rehabilitation facility was based on patients requiring inpatient level of care at the time of discharge in at least two different rehabilitation modalities: physical therapy, occupational therapy and/or speech therapy, as well as sufficient support mechanisms to allow for home discharge following the completion of the acute inpatient rehabilitation facility (AIRF) stay.

\section{Imaging analysis}

To evaluate potential tumor growth between surgical resection and initiation of chemoradiation, MRI scans were evaluated by a certified neuroradiologist [Author: AH]. All patients with excessive WT ( $>35$ days) with documented MRI scans within 1 week following surgical resection and within 1 week prior to treatment were evaluated. Patients were classified as having stable or increased contrast enhancement. When possible, measurements were taken according to Radiologic Assessment in Neuro-Oncology criteria [20].

\section{Statistical methods}

Patients were grouped according to their discharge disposition. Disposition groups included AIRF, skilled nursing facility/long-term care facility (SNF/LTC), home discharge with services (either home health services or outpatient therapies) or home discharge without services.

Nominal variables were described in absolute numbers and percentages for each discharge group. ASA classification was defined as a categorical variable, with patients divided into groups of ASA class $<3$ or $\geq 3$, as an ASA classification of 3 or higher indicates a patient with severe systemic disease. Nominal variables were compared between disposition group using the exact Chi-square test.

Age, LOS and WT are described within discharge groups using medians and ranges. Due to non-normally distributed data and/or unequal variances between groups, the Kruskal-Wallis test was used to assess differences 
Table 1. Summary of demographic information of each group based on disposition from hospital following initial surgery.

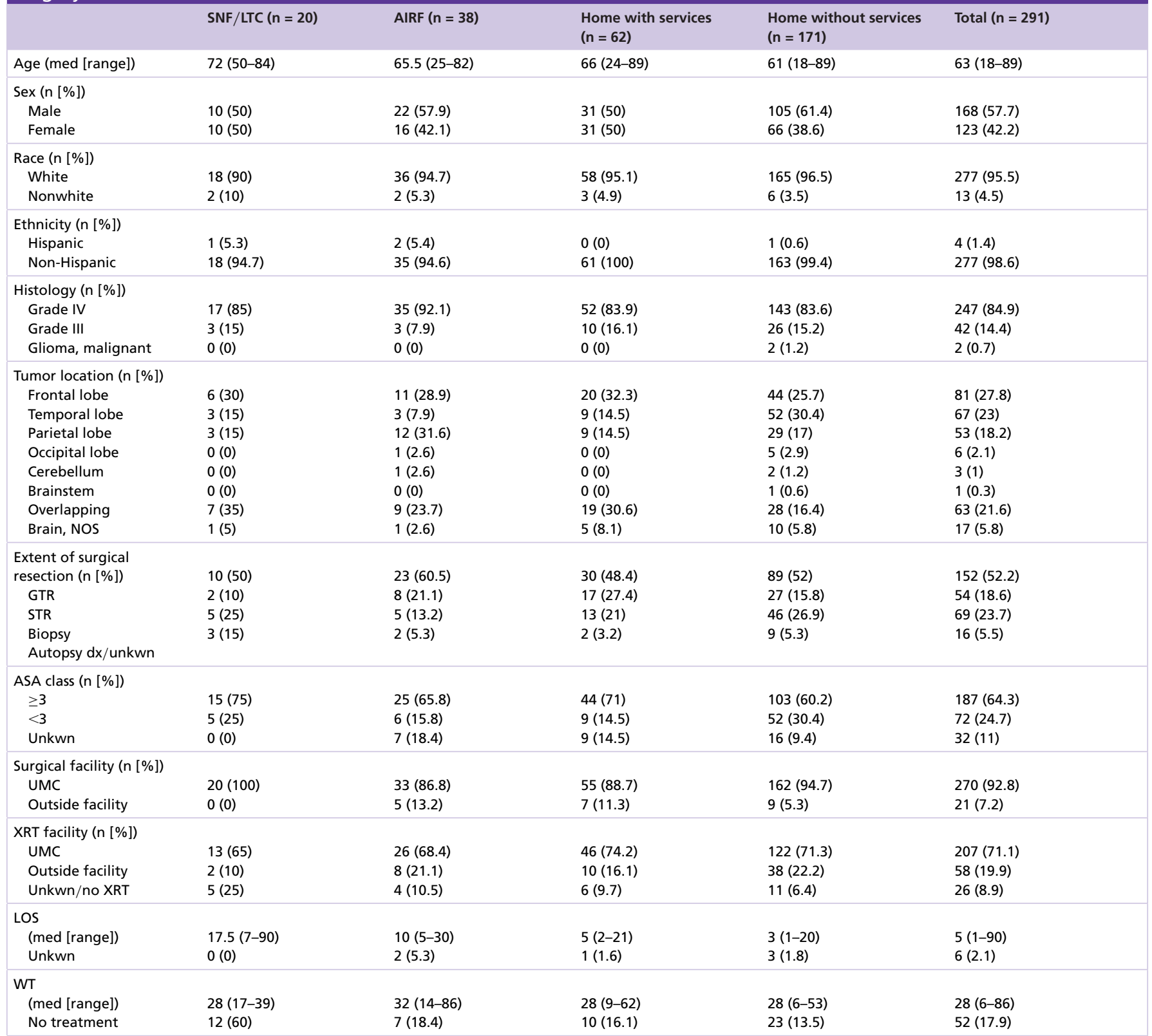

Information is also provided for the overall study population.

ASA: American Society of Anesthesiologists; AIRF: Acute inpatient rehabilitation facility; GTR: Gross total resection; LOS: Length of stay; LTC: Long-term care facility; NOS: Not otherwise specified; SNF: Skilled nursing facility; STR: Subtotal resection; UMC: University Medical Center; WT: Wait time; XRT: Radiation therapy.

between discharge groups. Post-hoc pairwise comparisons for these groups were performed using Wilcoxon rank sum test. Multiple comparison significance level was adjusted with the Bonferroni method. Excessive WT was defined as $>35$ days as 28 days \pm 1 week is often used as the standard of care at our institution. Odds ratio for an excessive WT was calculated using a logistic regression model for each disposition group with the home without services group used as the reference.

The best model for predicting WT was developed using least-squares regression. All factors in Table 1, except for LOS, were considered possible prognostic factors. Initially, each factor was evaluated in a univariate model. All factors with p-value $\leq 0.1$ were then tested jointly for association with WT in a multivariable regression model. Factors with a significance level $\leq 5 \%$ were considered independent predictors of WT. Estimated model coefficients 
$(\beta)$ represent the average change in WT (days) corresponding to factor levels. Since a major goal of this analysis was to test the effect of disposition on WT, all disposition groups were forced into the final regression model.

OS was estimated by the Kaplan-Meier method with differences pairwise comparisons assessed by a log-rank test. Analogous to the WT analysis, demographic factors were each evaluated for association with OS in univariate proportional hazards regression model. All factors with p-value $\leq 0.1$ were then tested for joint association with OS in a multivariable proportional hazard regression model. Because we have a specific aim to estimate the relationship between WT and disposition group with OS after controlling for other factors associated with OS, WT and all disposition groups were forced into the final model. For each variable, proportional hazards assumptions were tested using the Supremum Test with an alpha of 0.05 .

Subjects with missing or unknown data are excluded from analysis. It cannot be verified if this approach introduces bias, however, there is no known reason related to patient disposition that would affect data availability. Missing data is also described by discharge group (Table 1). All statistical analyses were performed using SAS v9.4 (SAS Institute, NC, USA). This manuscript was prepared including all recommended elements of the STROBE guidelines.

\section{Results}

Patient demographics

Of the 348 patients who were screened, 291 were included in this study: $84.9 \%$ with grade IV gliomas and $14.4 \%$ with grade III gliomas. Excluded patients were not included due to lack of information available in the electronic health record regarding disposition after surgical resection. Of these, 38 patients were admitted to AIRF, 20 patients were discharged to SNF/LTC, 62 patients were discharged home with services and 171 patients were discharged to home without services. The cohort included 168 males and 123 females, with $95.5 \%$ of subjects identifying as Caucasian and $98.6 \%$ with non-Hispanic ethnicity. Median age at diagnosis was 63 (range: 18-89). Initial surgical procedures included gross total resection (52.2\%), subtotal resection (18.6\%) and biopsy only (23.7\%). A total of $64.3 \%$ of patients were classified as ASA $\geq 3$. The most common tumor location was in the frontal lobe (27.8\%), followed by temporal lobe (23\%) and parietal lobe (18.2\%). Demographic information was also reported separately for each discharge group (Table 1). On Chi-square test, differences were only found in the proportion of patients with temporal tumor location $(\mathrm{p}=0.0037)$ and overlapping tumor location $(\mathrm{p}=0.0457)$.

\section{Length of stay analysis}

Median LOS was: 3 days (range: 1-20) for patients discharged to home without services, 5 days (range: $0-21$ ) for patients discharged home with services, 10 days (range: 5-30) for patients discharged to AIRF and 17.5 days (range: 7-90) for patients discharged to SNF/LTC (Figure 1A). Kruskal-Wallis test indicated a significant association between discharge group and LOS $\left(\mathrm{X}^{2}=127.5, \mathrm{p}<0.0001\right)$. Wilcoxon rank sum comparison of individual groups revealed a statistically significant difference in LOS between every group with $\mathrm{p}<0.0001$.

\section{Wait time analysis}

Median WT for patients discharged to home without services was 28 days (range: 6-53), to home with services was 28 days (range: 9-62), to AIRF was 32 days (range: 14-86) and to SNF/LTC was 28 days (range: 17-39) (Figure 1B).

WT was significantly different between discharge groups using Kruskal-Wallis test $\left(\mathrm{X}^{2}=12.2026, \mathrm{p}=0.0067\right)$. Wilcoxon rank sum pairwise comparison revealed a statistically significant difference in WT between AIRF patients compared with home discharge without services patients $\left(X^{2}=10.64, p=0.0011\right)$. All other pairwise comparisons were not statistically significant $(\mathrm{p}>0.05)$.

Patients admitted to AIRF were found to have significantly higher odds of excessive WT compared with home discharge without services patients, with an odds ratio of 3.13 (95\% CI: 1.38-7.08, $\mathrm{p}=0.0063$ ) as well as patients discharged home with services compared with patients discharged home without services (OR: 2.16, 95\% CI: 1.04-4.50, $\mathrm{p}=0.0395)$. No significant difference was found in excessive WT for SNF/LTC patients compared with patients discharged to home without services.

Using univariate regression analysis, the following demographic factors were associated with either prolonged or shortened WT at the $10 \%$ significance level: female sex $(\beta=-2.433, \mathrm{p}=0.0613)$, biopsy only $(\beta=-2.85, \mathrm{p}=0.0785)$, surgery at an outside surgical facility $(\beta=7.615, \mathrm{p}=0.0007)$, discharge to $\operatorname{AIRF}(\beta=7.318, \mathrm{p}=0.0002)$, discharge home with services $(\beta=3.841, \mathrm{p}=0.0143)$ and radiation treatment at an outside facility $(\beta=-2.727, \mathrm{p}=0.0776)$. 
(A)

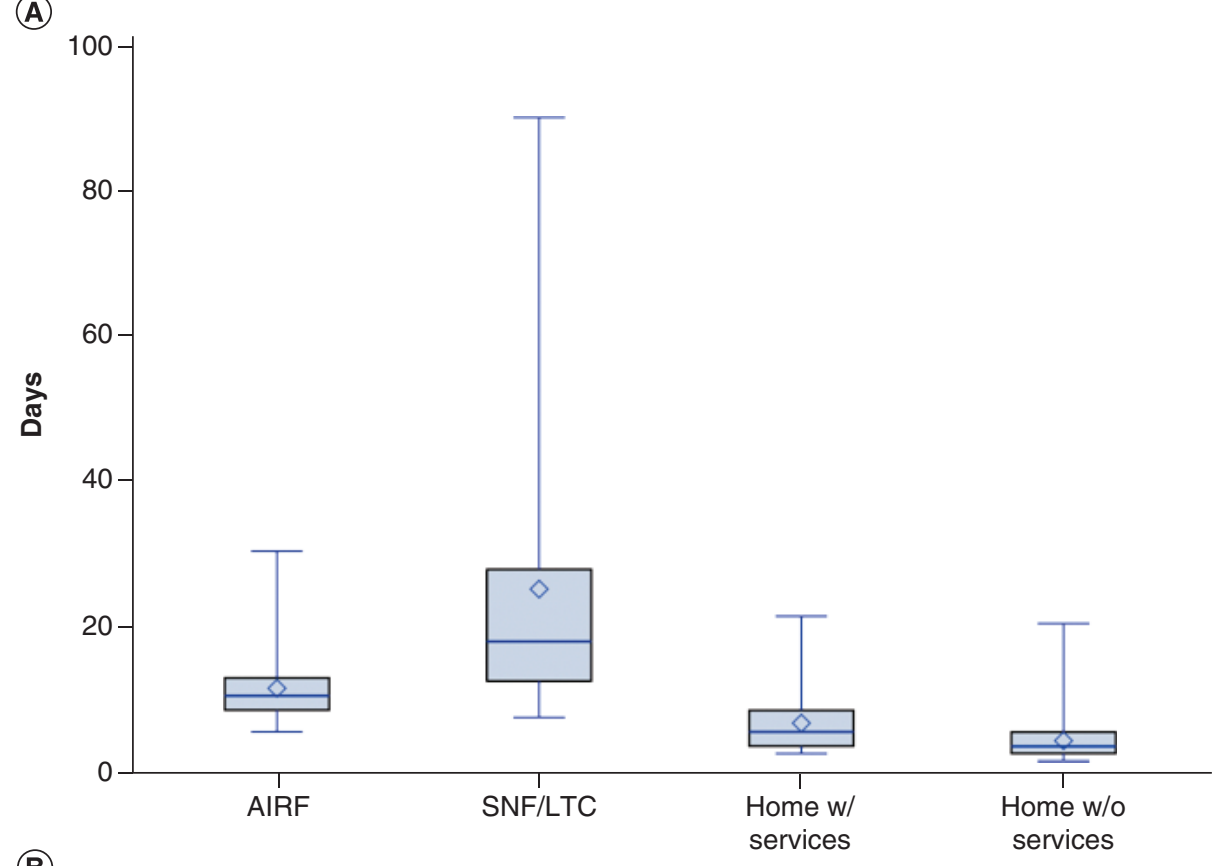

(B)

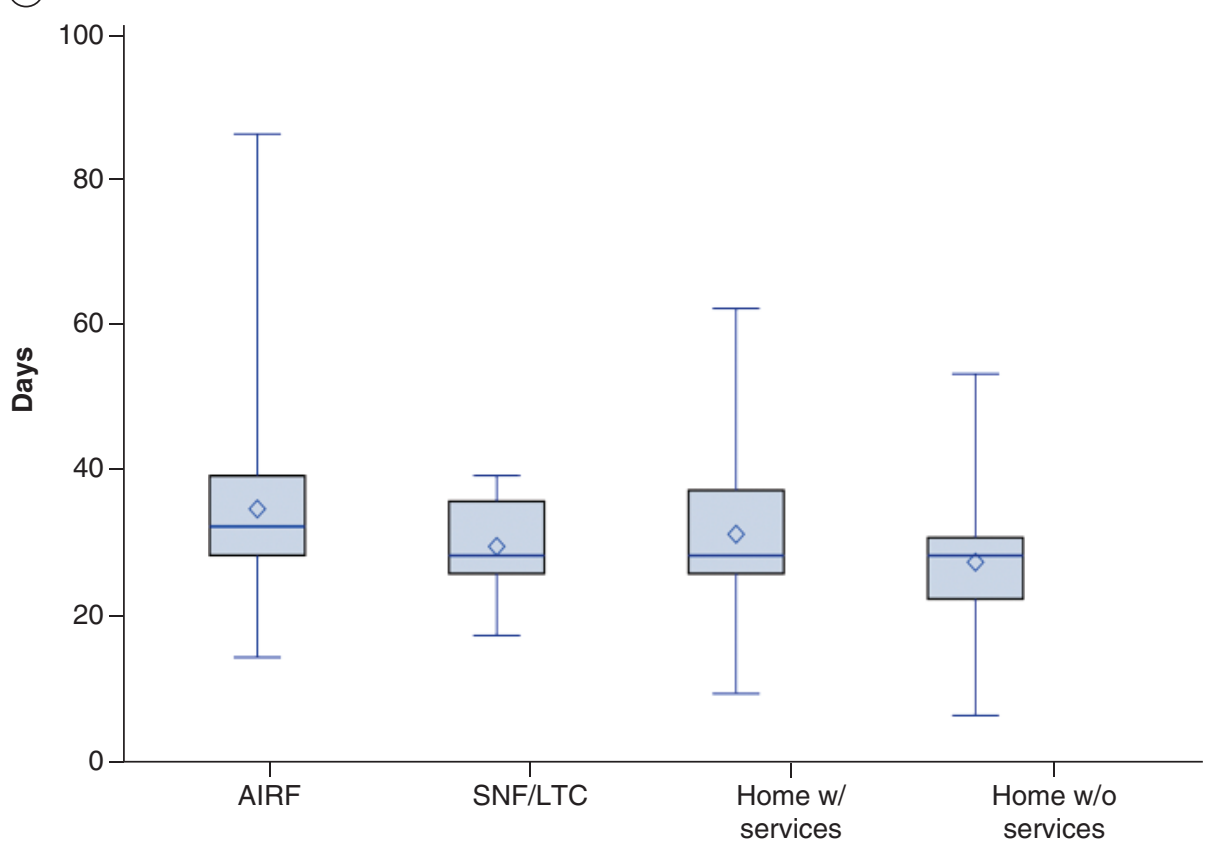

Figure 1. Length of hospital stay and wait time for high-grade glioma patients by disposition group. (A) Box plot depicting the length of the hospital stay in which patients had their first surgical resection/biopsy. (B) Box plot depicting time between surgery and initiation of chemoradiation for each discharge group.

Bars represent maximum and minimum values. Lines forming the boxes represent 3rd quartile, median and 1st quartile values (from top to bottom). symbol indicates mean values in each group.

AIRF: Acute inpatient rehabilitation facility; LTC: Long-term care facility; SNF: Skilled nursing facility.

On multivariable analysis sex, biopsy only, outside surgical facility, discharge to AIRF and discharge home with services, remained significant (Table 2). 


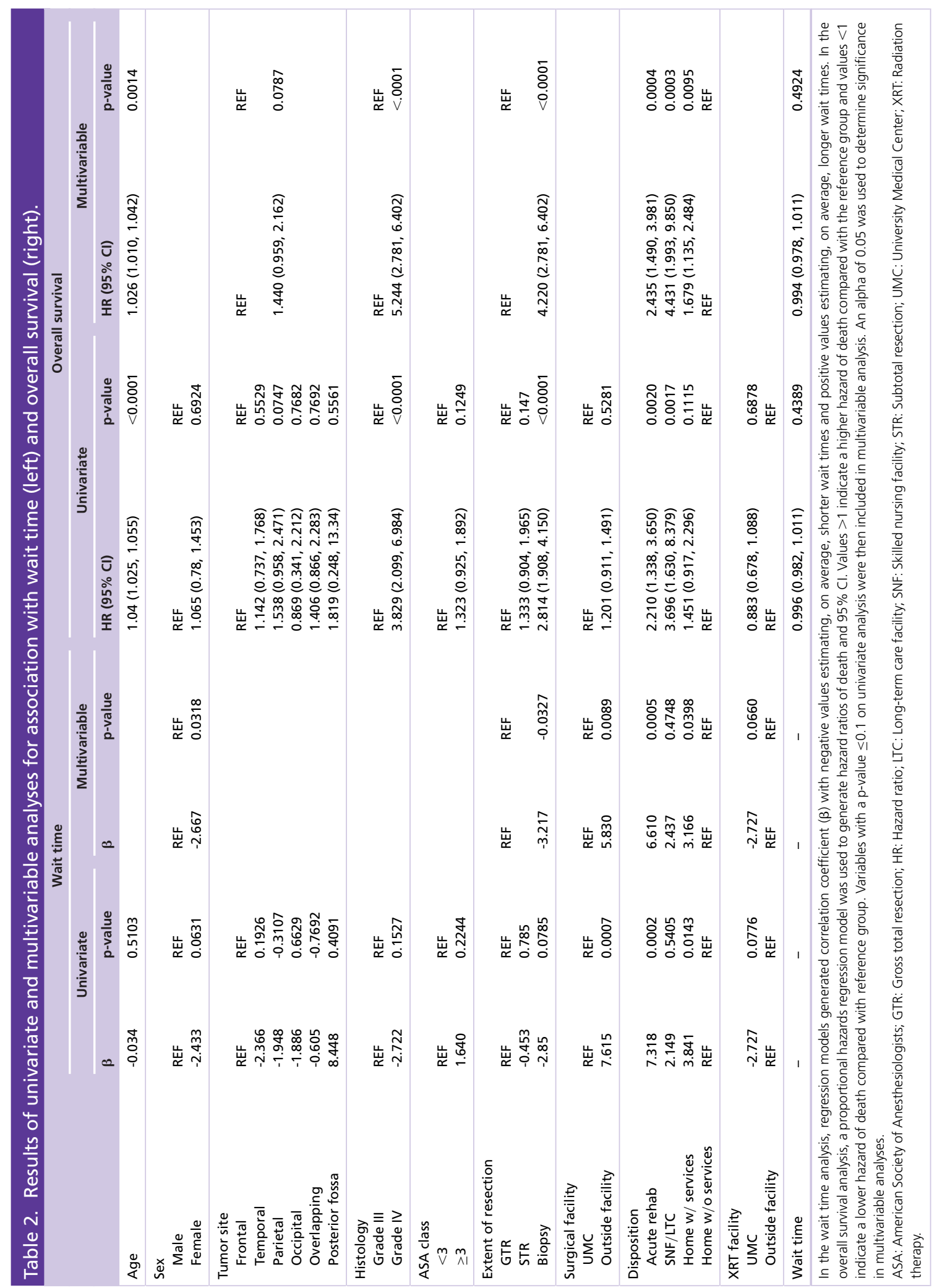




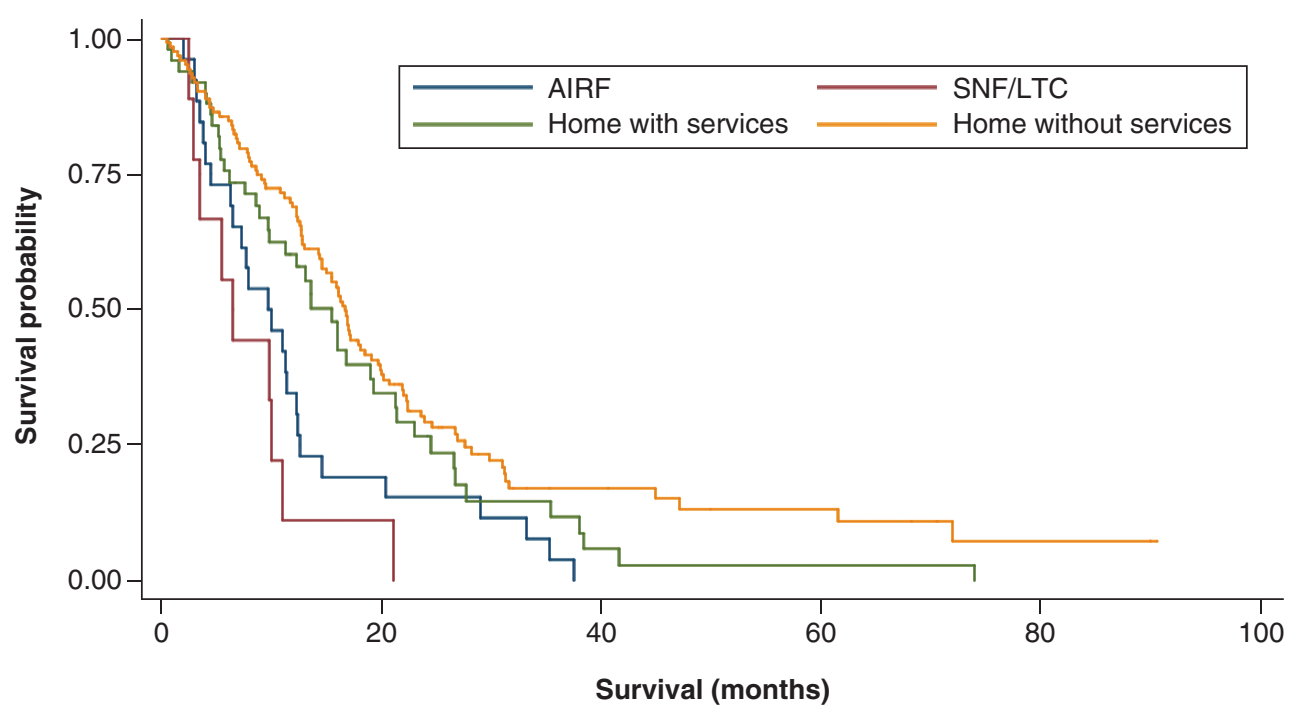

Figure 2. Kaplan-Meier survival curves depicting overall survival for each group. In this cohort, overall survival was defined starting at time of initiation of chemoradiation until date of death/last date of follow-up.

AIRF: Acute inpatient rehabilitation facility; LTC: Long-term care facility; SNF: Skilled nursing facility.

\section{Survival analysis}

Median OS for patients discharged home without services was 16.7 months (95\% CI: 14.6-19.1), home with services was 15.5 months (95\% CI: 9.7-19.3), to AIRF was 9.8 months (95\% CI: 6.3-12.3) and to SNF/LTC was 6.5 months (95\% CI: 2.5-11.0). Discharge destination was significantly associated with OS, unadjusted for other patient characteristics, using the log-rank test $\left(\mathrm{X}^{2}=21.36, \mathrm{p}<0.0001\right)$. Pairwise analysis revealed significant differences in OS between AIRF and home without services patients $\left(\mathrm{X}^{2}=12.09, \mathrm{p}=0.003\right)$ and between SNF/LTC and home without services patients $\left(\mathrm{X}^{2}=13.85, \mathrm{p}=0.0012\right)$. No other pairwise comparisons yielded statistically significant results (Figure 2).

A proportional hazards regression model revealed no significant association between WT and OS (HR: 0.996; $\mathrm{p}=0.4389)$. Demographic factors significantly associated at the $10 \%$ significance level with decreased OS on univariate analysis included younger age (HR: 1.04, p < 0.0001), parietal tumor location (HR: 1.538, p =0.0747), grade IV histology (HR: 3.829, p < 0.0001), biopsy only (HR: 2.814, p < 0.0001), discharge to AIRF (HR: 2.21, $\mathrm{p}=0.002)$ and discharge to SNF/LTC (HR: 3.696, $\mathrm{p}=0.0017)$. In multivariable analysis, age, grade IV histology, biopsy only, discharge to AIRF and discharge to SNF/LTC remained significant. Even after adjusting for each of these demographic factors, WT was still not significantly associated with OS (HR: 0.994, p = 0.4924) (Table 2).

\section{Imaging analysis}

Of the 44 subjects with excessive WT, 12 (27\%) of them had MRI scans within 1 week following surgery and within 1 week prior to chemoradiation that were available. Of these patients, $66.7 \%$ were noted to have an increase in contrast enhancement during this interval. Among the patients with measurable lesions, the mean change in contrast-enhanced area was $77.0 \mathrm{~mm}^{2}$ (Figure 3).

\section{Discussion}

This study indicates that there may be differences in treatment and outcomes for patients with HGGs based on discharge location following resection. Compared with patients who were discharged home, patients who required any inpatient rehabilitation were found to have a longer LOS, longer WT, higher odds of excessive treatment delay and shorter OS when examined separately. Although the median differences between AIRF and home discharge patients was minimal (32 vs 28 days), which may have little clinical significance, the fact that patients admitted to rehabilitation facilities have a much greater odds of an excessive delay in treatment is the most relevant to treatment timing in these patients. These results are consistent with our hypotheses: a longer LOS would be expected for these patients, as they must be evaluated by physical and occupational therapists, accepted to a rehabilitation program and receive insurance approval prior to discharge [21]. Indeed, in this cohort, LOS was found to be significantly higher 


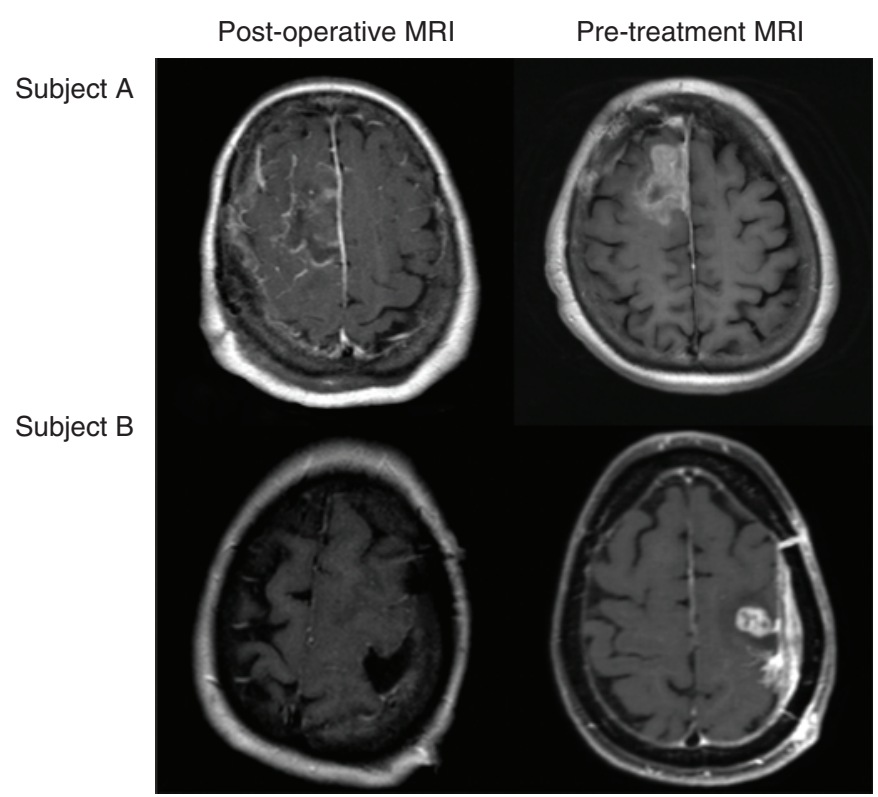

Figure 3. MRI depicting changes in tumor size in subject $A$ (top) and subject B (bottom). The images on the left were obtained within 1 week of surgical resection and images on the right were obtained within 1 week prior to the initiation of chemoradiation. For subject $A$, the increase in contrast enhancement during this time interval was determined to be $118.55 \mathrm{~mm}^{2}$, and for subject $\mathrm{B}$, it was 79.55 $\mathrm{mm}^{2}$.

for patients evaluated by physical therapy independent of disposition $(\mathrm{p}<0.0001)$. Longer LOS is associated with many well-known risks including hospital-acquired infections, increased overall mortality and increased healthcare costs [22]. Additionally, as patients are often unable to receive cancer treatment until rehabilitation is complete, a longer WT would also be expected. In our experience, some rehabilitation facilities will not accept patients who are going to receive chemotherapy at all and these patients are treated with radiation alone as a result. While this decision is in part based on the patient's performance status, there is some bias based on insurance reimbursement policies. It is an interesting finding that patients who are discharged to AIRF have longer WT than patients discharged to SNF/LTC facilities. This could be due in part to the fact that SNF and LTC facilities are designed for longer term care and may not have the same restrictions on the types of treatments patients are able to receive. In addition to disposition, the discrepancy in OS could be due to a number of factors that have been shown to influence outcomes in HGG, such as functional status, that are not comprehensively explored [23-25].

While this study did not show an association between WT and OS, previous studies with robust analyses have demonstrated varying relationships [10,12,19,26-32]. Pollom et al. conducted a large retrospective cohort study of over 12,000 patients to evaluate the optimal timing of treatment initiation in GBM patients and discovered an inverse relationship between WT and OS, particularly in patients who underwent gross total resection [28]. Unlike our present study, Pollom et al. had a large sample size and created a model for assessing the relationship between WT and OS that controlled for several important prognostic factors, including age, sex, race and comorbidity scoring. Recent studies that have not found a relationship between WT and OS have comparatively smaller sample sizes and/or fail to control for other prognostic factors [12,26,30,31]. Naturally, there is concern that leaving such an aggressive tumor with a rapid doubling time untreated for several weeks could allow for further tumor growth [33]. Studies have shown increased contrast enhancement between the time of tumor resection and therapy initiation consistent with tumor progression in over $80 \%$ of patients, which is consistent with the imaging results found in this study [34]. Moreover, it has been shown that patients with radiological evidence of tumor progression prior to chemoradiation have decreased survival when compared with patients without evidence of tumor progression [35]. Due to ethical concerns, no prospective study has been designed to define the relationship between WT and prognosis. Despite the absence of conclusive data, the prospect of a delay in treatment of HGG can nevertheless serve as a source of anxiety for patients, family and providers. However, in support of a generally accepted oncological principle, patients who are physically stronger before cancer treatment will survive longer. This is why other studies have shown that increasing a person's physical strength before beginning intensive chemotherapy or radiation therapy can lead to better outcomes. The worse OS in patients discharged to rehabilitation is likely a reflection of the poorer performance status of these patients, though it is hard to imagine that delaying the treatment of these patients would be beneficial. 
This study has several limitations. The single-institution, retrospective design has several disadvantages including the possibility of selection bias and questionable generalizability. Furthermore, the data that were collected were dependent upon prior record keeping and some factors that may have been informative in this analysis, such as functional status/comorbidity scoring and neurological status, were not consistently available. This study also had a relatively small number of rehabilitation patients that make it difficult to draw definitive conclusions about these populations. Also, in New York state, long term acute care facilities are not permitted, which limits the generalizability to other states whose postacute care options are more available. Third party payer policies and the presence or absence of insurance coverage itself often play the determining role in whether a patient is eligible for discharge to an AIRF or LTC/SNF. As a retrospective cohort study, our study is limited in that there is no way to capture how much of an impact those external forces may have had in patient disposition for our population as a whole. Such decisions are made on a case-by-case basis and are rarely completely documented in the medical record.

As the benefits of rehabilitation programs on the functional status, quality of life and prognosis of patients with brain tumors have been well described [4-7,9], it is important to identify any factors that may diminish these benefits or otherwise serve as a disincentive for optimal treatment of patients who may benefit from rehabilitation. Defining this disparity in treatment and outcomes is an essential first step in mitigating this discrepancy. This is especially true as patients who are discharged to rehabilitation locations often have worse functional status which is, in itself, a negative prognostic factor. Indeed, patients admitted to any rehabilitation facility in this cohort had older age and higher ASA scores than patients who were discharged home, which also impact disease outcomes. Intraoperative implantation of carmustine wafers is a potential bridge therapy option for patients who are expected to have delayed treatment due to rehabilitation, though it is difficult to identify these patients pre-operatively. While this study is the first to provide evidence of treatment discrepancies based on discharge placements in HGG, more comprehensive, larger scale, multicenter studies should be conducted to corroborate these findings and minimize regional bias.

\section{Conclusion}

HGG patients who are discharged to rehabilitation facilities following initial surgery have longer LOS, longer WT and shorter OS compared with patients who are discharged home. These results are important to consider when counseling patients and families regarding treatment decisions, as discharge location in itself may be an important contributor to patient outcomes. Ultimately, the goal is to focus on creating protocols that allow HGG patients to receive the full benefits of rehabilitation without the prospect of treatment delays or unfavorable outcomes.

\section{Practice points}

- Patients with high-grade glioma who are discharged to rehabilitation facilities following surgery wait longer for treatment initiation than those who are discharged home.

- Patients with high-grade glioma who are discharged to rehabilitation facilities have longer hospital stay and worse outcomes than those who are discharged home.

Supplementary data

To view the supplementary data that accompany this paper please visit the journal website at: www.futuremedicine.com/doi/suppl/10.2217/cns-2020-0018

\section{Author contributions}

KT Warren collected data, performed statistical analyses and wrote the manuscript. L Liu assisted with statistical analyses and data representation and provided valuable feedback on the manuscript. Y Liu collected some data and provided valuable feedback on the manuscript. MS Strawderman provided guidance and verification of all statistical analyses as well as valuable feedback on the manuscript. AH Hussain performed imaging analysis and provided valuable feedback on the manuscript. HM Ma provided expertise in physical medicine and rehabilitation as well as valuable feedback on the manuscript. MT Milano provided expertise in radiation oncology as well as valuable feedback on the manuscript. NA Mohile provided expertise in neuro-oncology as well as valuable feedback on the manuscript. KA Walter generated the idea for this study, guided the primary author throughout the process and provided valuable feedback on the manuscript. 
Financial \& competing interests disclosure

The project described in this publication was supported by the University of Rochester Clinical \& Translational Science Award TL1 TR002000 from the National Center for Advancing Translational Sciences of the National Institutes of Health. The content is solely the responsibility of the authors and does not necessarily represent the official views of the National Institutes of Health. The authors have no other relevant affiliations or financial involvement with any organization or entity with a financial interest in or financial conflict with the subject matter or materials discussed in the manuscript apart from those disclosed.

No writing assistance was utilized in the production of this manuscript.

Ethical conduct of research

All procedures performed in studies involving human participants were in accordance with the ethical standards of the University of Rochester Research Subject Review Board (RSRB00072421) and with the 1964 Helsinki declaration and its later amendments or comparable ethical standards. Information regarding participants in this study was obtained with a waiver of informed consent.

Open access

This work is licensed under the Attribution-NonCommercial-NoDerivatives 4.0 Unported License. To view a copy of this license, visit http://creativecommons.org/licenses/by-nc-nd/4.0/

\section{References}

Papers of special note have been highlighted as: $\bullet$ of interest; $\bullet \bullet$ of considerable interest

1. Ostrom QT, Gittleman H, Fulop J et al. CBTRUS statistical report: primary brain and central nervous system tumors diagnosed in the United States in 2008-2012. Neuro Oncol. 17(Suppl. 4), iv1-iv62 (2015).

2. Stupp R, Mason WP, van den Bent MJ et al. Radiotherapy plus concomitant and adjuvant temozolomide for glioblastoma. N. Engl. J. Med. 352(10), 987-996 (2005).

-. This article is the first to describe the survival benefit with the current standard of care treatment for high-grade glioma: maximum safe surgical resection followed by concurrent radiation and temozolomide treatment.

3. Lakomkin N, Hadjipanayis CG. Non-routine discharge disposition is associated with post-discharge complications and 30-day readmissions following craniotomy for brain tumor resection. J. Neurooncol. 136(3), 595-604 (2018).

4. Tang V, Rathbone M, Park Dorsay J, Jiang S, Harvey D. Rehabilitation in primary and metastatic brain tumours: impact of functional outcomes on survival. J. Neurol. 255(6), 820-827 (2008).

- Describes the benefit of inpatient rehabilitation for patients with brain tumors, both primary and metastatic, in both functional outcomes and overall survival.

5. Marciniak CM, Sliwa JA, Heinemann AW, Semik PE. Functional outcomes of persons with brain tumors after inpatient rehabilitation. Arch. Phys. Med. Rehabil. 82(4), 457-463 (2001).

6. Huang ME, Wartella JE, Kreutzer JS. Functional outcomes and quality of life in patients with brain tumors: a preliminary report. Arch Phys. Med. Rehabil. 82(11), 1540-1546 (2001).

7. O'Dell MW, Barr K, Spanier D, Warnick RE. Functional outcome of inpatient rehabilitation in persons with brain tumors. Arch. Phys. Med. Rehabil. 79(12), 1530-1534 (1998).

8. Fu JB, Parsons HA, Shin KY et al. Comparison of functional outcomes in low- and high-grade astrocytoma rehabilitation inpatients. Am. J. Phys. Med. Rehabil. 89(3), 205-212 (2010).

9. Huang ME, Sliwa JA. Inpatient rehabilitation of patients with cancer: efficacy and treatment considerations. PMR 3(8), 746-757 (2011).

10. Han SJ, Rutledge WC, Molinaro AM et al. The effect of timing of concurrent chemoradiation in patients with newly diagnosed glioblastoma. Neurosurgery 77(2), 248-253 (2015).

11. Lai R, Hershman DL, Doan T, Neugut AI. The timing of cranial radiation in elderly patients with newly diagnosed glioblastoma multiforme. Neuro Oncol. 12(2), 190-198 (2010).

12. Noel G, Huchet A, Feuvret L et al. Waiting times before initiation of radiotherapy might not affect outcomes for patients with glioblastoma: a French retrospective analysis of patients treated in the era of concomitant temozolomide and radiotherapy. $J$. Neurooncol. 109(1), 167-175 (2012).

13. Lutterbach J, Weigel P, Guttenberger R, Hinkelbein W. Accelerated hyperfractionated radiotherapy in 149 patients with glioblastoma multiforme. Radiother. Oncol. 53(1), 49-52 (1999).

14. Irwin C, Hunn M, Purdie G, Hamilton D. Delay in radiotherapy shortens survival in patients with high grade glioma. J. Neurooncol. 85(3), 339-343 (2007).

15. Glinski B, Urbański J, Hetnał $\mathrm{M}$ et al. Prognostic value of the interval from surgery to initiation of radiation therapy in correlation with some histo-clinical parameters in patients with malignant supratentorial gliomas. Contemp. Oncol. (Pozn.) 16(1), 34-37 (2012).

16. Do V, Gebski V, Barton M. The effect of waiting for radiotherapy for grade III/IV gliomas. Radiother. Oncol. 57(2), 131-136 (2000). 
17. Blumenthal DT, Won M, Mehta MP et al. Short delay in initiation of radiotherapy may not affect outcome of patients with glioblastoma: a secondary analysis from the radiation therapy oncology group database. J. Clin. Oncol. 27(5), 733-739 (2009).

18. Wehming FM et al. Malignant glioma grade 3 and 4: how relevant is timing of radiotherapy? Clin. Neurol. Neurosurg. 114(6), 617-621 (2012).

19. Nathan JK, Brezzell AL, Kim MM, Leung D, Wilkinson DA, Hervey-Jumper SL. Early initiation of chemoradiation following index craniotomy is associated with decreased survival in high-grade glioma. J. Neurooncol. 135(2), 325-333 (2017).

20. Wen PY, Macdonald DR, Reardon DA et al. Updated response assessment criteria for high-grade gliomas: response assessment in neuro-oncology working group. J. Clin. Oncol. 28(11), 1963-1972 (2010).

21. New PW, Andrianopoulos N, Cameron PA, Olver JH, Stoelwinder JU. Reducing the length of stay for acute hospital patients needing admission into inpatient rehabilitation: a multicentre study of process barriers. Intern. Med. J. 43(9), 1005-1011 (2013).

22. Rosman M, Rachminov O, Segal O, Segal G. Prolonged patients' In-Hospital Waiting Period after discharge eligibility is associated with increased risk of infection, morbidity and mortality: a retrospective cohort analysis. BMC Health Serv. Res. 15, 246 (2015).

23. Mazaris P, Hong X, Altshuler D et al. Key determinants of short-term and long-term glioblastoma survival: a 14-year retrospective study of patients from the Hermelin Brain Tumor Center at Henry Ford Hospital. Clin. Neurol. Neurosurg. 120, 103-112 (2014).

24. Brown TJ, Brennan MC, Li M et al. Association of the extent of resection with survival in glioblastoma: a systematic review and meta-analysis. JAMA Oncol. 2(11), 1460-1469 (2016).

25. Ma X, Lv Y, Liu J et al. Survival analysis of 205 patients with glioblastoma multiforme: clinical characteristics, treatment and prognosis in China. J. Clin. Neurosci. 16(12), 1595-1598 (2009).

26. Blumenthal DT, Won M, Mehta MP et al. Short delay in initiation of radiotherapy for patients with glioblastoma-effect of concurrent chemotherapy: a secondary analysis from the NRG Oncology/Radiation Therapy Oncology Group database. Neuro. Oncol. 20(7), 966-974 (2018).

27. Adeberg $\mathrm{S}$, Bostel $\mathrm{T}$, Harrabi $\mathrm{S}$ et al. Impact of delays in initiating postoperative chemoradiation while determining the MGMT promoter-methylation statuses of patients with primary glioblastoma. BMC Cancer 15, 558 (2015).

28. Pollom EL, Fujimoto DK, Han SS et al. Newly diagnosed glioblastoma: adverse socioeconomic factors correlate with delay in radiotherapy initiation and worse overall survival. J. Radiat. Res. 59(Suppl. 1), i11-i18 (2018).

-. Describes a large review of over 12,000 patients with glioblastoma and demonstrated an inverse relationship between wait time to start treatment and overall survival, especially in patients who undergo gross total resection after controlling for several important demographic factors.

29. Sun MZ, Oh T, Ivan ME et al. Survival impact of time to initiation of chemoradiotherapy after resection of newly diagnosed glioblastoma. J. Neurosurg. 122(5), 1144-1150 (2015).

30. Louvel G, Metellus P, Noel G et al. Delaying standard combined chemoradiotherapy after surgical resection does not impact survival in newly diagnosed glioblastoma patients. Radiother. Oncol. 118(1), 9-15 (2016).

31. Osborn VW, Lee A, Garay E, Safdieh J, Schreiber D. Impact of timing of adjuvant chemoradiation for glioblastoma in a large hospital database. Neurosurgery 83(5), 915-921 (2018).

32. Wang TJ, Jani A, Estrada JP et al. Timing of adjuvant radiotherapy in glioblastoma patients: a single-institution experience with more than 400 patients. Neurosurgery 78(5), 676-682 (2016).

33. Kirkby NF, Jefferies SJ, Jena R, Burnet NG. A mathematical model of the treatment and survival of patients with high-grade brain tumours. J. Theor. Biol. 245(1), 112-124 (2007).

34. Farace P, Amelio D, Ricciardi GK et al. Early MRI changes in glioblastoma in the period between surgery and adjuvant therapy. $J$. Neurooncol. 111(2), 177-185 (2013).

35. Pirzkall A, McGue C, Saraswathy $S$ et al. Tumor regrowth between surgery and initiation of adjuvant therapy in patients with newly diagnosed glioblastoma. Neuro Oncol. 842-852 (2009).

- Demonstrates that high-grade glioma patients who show evidence of radiographic disease progression prior to initiating chemoradiation have worse outcomes. 